\title{
Enhanced Corrosion Resistance and Cytocompatibility of Biomimetic Hyaluronic Acid Functionalised Silane Coating on AZ31 Mg Alloy for Orthopaedic Applications
}

\author{
Sankalp Vinod Agarwal \\ Technological University Dublin, Centre for Research in Engineering Surface Technology (CREST), Dublin, \\ Ireland \\ Marie Noëlle Labour \\ Institut Galilee, Villetaneuse, France \\ David A. Hoey \\ Trinity College Dublin, Trinity Centre for Bioengineering, Dublin, Ireland
}

See next page for additional authors

Follow this and additional works at: https://arrow.tudublin.ie/scschcpsart

Part of the Medicine and Health Sciences Commons

\section{Recommended Citation}

Agarwal, S., Labour, M.N. \& Hoey, D. (2018). Enhanced Corrosion Resistance and Cytocompatibility of Biomimetic Hyaluronic Acid Functionalised Silane Coating on AZ31 Mg Alloy for Orthopaedic Applications. Journal of Materials Science, vol. 29, no. 9, article number 144. Doi:10.1007/ s10856-018-6150-5

This Article is brought to you for free and open access by the School of Chemical and Pharmaceutical Sciences at ARROW@TU Dublin. It has been accepted for inclusion in Articles by an authorized administrator of ARROW@TU Dublin. For more information, please contact arrow.admin@tudublin.ie, aisling.coyne@tudublin.ie, gerard.connolly@tudublin.ie.

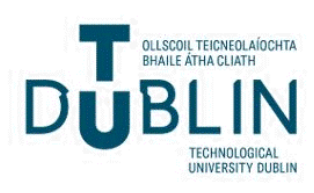




\section{Authors}

Sankalp Vinod Agarwal, Marie Noëlle Labour, David A. Hoey, Brendan Duffy, James Curtin, and Swarna Jaiswal

This article is available at ARROW@TU Dublin: https://arrow.tudublin.ie/scschcpsart/83 


\title{
Enhanced corrosion resistance and cytocompatibility of biomimetic hyaluronic acid functionalised silane coating on AZ31 Mg alloy for orthopaedic applications
}

\author{
Sankalp Agarwal ${ }^{1,2} \cdot$ Marie-Noelle Labour $^{3,4} \cdot$ David Hoey $^{3,4,5} \cdot$ Brendan Duffy $^{1} \cdot$ James Curtin $^{2} \cdot$ Swarna Jaiswal $^{1}$
}

Received: 4 January 2018 / Accepted: 12 August 2018

(c) Springer Science+Business Media, LLC, part of Springer Nature 2018

\begin{abstract}
This paper reports the corrosion resistant and cytocompatible properties of the hyaluronic acid-silane coating on AZ31 Mg alloy. In this study, the osteoinductive properties of high molecular weight hyaluronic acid (HA, 1-4 MDa) and the corrosion protection of silane coatings were incorporated as a composite coating on biodegradable AZ31 Mg alloy for orthopaedic applications. The multi-step fabrication of coatings first involved dip coating of a passivated AZ31 Mg alloy with a methyltriethoxysilane-tetraethoxysilane sol-gel to deposit a dense, cross-linked and corrosion resistant silane coating (AZ31MT). The second step was to create an amine-functionalised surface by treating coated alloy with 3-aminopropyl-triethoxy silane (AZ31-MT-A) which facilitated the immobilisation of HA via EDC-NHS coupling reactions at two different concentrations i.e $1 \mathrm{mg} \cdot \mathrm{ml}^{-1}$ (AZ31-MT-A-HA1) and $2 \mathrm{mg}^{-\mathrm{ml}^{-1}}$ (AZ31-MT-A-HA2). These coatings were characterised by Fourier transform infrared spectroscopy, atomic force microscopy and static contact angle measurements which confirmed the successful assembly of the full coatings onto AZ31 Mg alloy. The influence of HA-silane coating on the corrosion of Mg alloy was investigated by electrical impedance spectroscopy and long-term immersion studies measurements in HEPES buffered DMEM. The results showed an enhanced corrosion resistance of HA functionalised silane coated AZ31 substrate over the uncoated equivalent alloy. Furthermore, the cytocompatibility of MC3T3-E1 osteoblasts was evaluated on HAcoated AZ31-MT-A substrates by live-dead staining, quantification of total cellular DNA content, scanning electron microscope and alkaline phosphatase activity. The results showed HA concentration-dependent improvement of osteoblast cellular response in terms of enhanced cell adhesion, proliferation and differentiation. These findings hold great promise in employing such biomimetic multifunctional coatings to improve the corrosion resistance and cytocompatibility of biodegradable Mg-based alloy for orthopaedic applications.
\end{abstract}

Electronic supplementary material The online version of this article (https://doi.org/10.1007/s10856-018-6150-5) contains supplementary material, which is available to authorized users.

Swarna Jaiswal

swarna.jaiswal@dit.ie

swarna.jaiswal@outlook.com

1 Centre for Research in Engineering and Surface Technology, Dublin Institute of Technology, Kevin Street, Dublin 8, Ireland

2 School of Food Science and Environmental Health, Cathal Brugha Street, Dublin Institute of Technology, Dublin 1, Ireland
3 Trinity Centre for Bioengineering, Trinity Biomedical Sciences Institute, Trinity College Dublin, Dublin 2, Ireland

4 Department of Mechanical and Manufacturing Engineering, School of Engineering, Trinity College Dublin, Dublin 2, Ireland

5 Advanced Materials and Bioengineering Research Centre, Royal College of Surgeons in Ireland and Trinity College Dublin, Dublin 2, Ireland 


\section{Graphical Abstract}

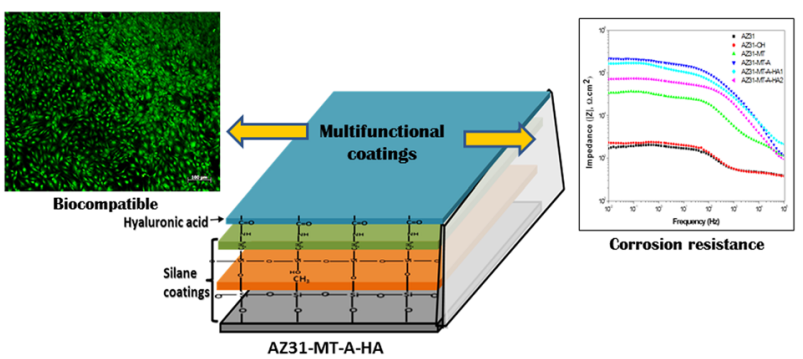

\section{Introduction}

The most widely used metals for orthopaedic implants are stainless steel, titanium and its alloys due to their strength and chemical inertness [1]. Due to these physical features, the mechanical properties of these metals mismatch with the natural bone and several complications such as an inflammatory response, physical irritation or stress shielding may occur after surgery [1]. As an alternative, biodegradable metals have received great interest due to their ability to provide structural support for an initial period following insertion. Following a suitable dwell time, during which the body recovers, the implant begins to degrade, resulting in a strengthened bone structure. Magnesium (Mg) based biodegradable alloys have such osteoinductive properties in combination with excellent physical and mechanical properties [2]. These properties make Mg-based alloys suitable for the fixation of bone fractures through plates, rods, and screws. To date, the major difficulty hampering their clinical application is the rapid degradation in physiological conditions [3]. This results in the elevated evolution of hydrogen gas which leads to inflammation, especially during the early stages of implantation [3].

Several approaches have been investigated to control the degradation of $\mathrm{Mg}$ alloys and improve the biocompatibility. Generally, the selective use of alloying elements has improved the mechanical and corrosion resistance properties of $\mathrm{Mg}$ alloys [4]. However, alloying elements may cause potential cellular toxicities. Alternatively, surface modifications such as micro-arc oxidation and ion implantation have been studied as potential methods to improve the corrosion resistance of $\mathrm{Mg}$ alloy [5]. However, these surface modifications are unable to resist long-term corrosion protection under in vivo conditions [5]. Therefore, surface coatings are now being investigated as an effective approach with the potential to improve the corrosion resistance and biocompatibility of $\mathrm{Mg}$ alloys.

Amongst surface coatings, organosilane-based chemistry has been widely employed due to their chemical stability and effective corrosion protection on lightweight metals [6].
Organosilanes possess functional organic and hydrolysable alkoxy groups [7]. Upon hydrolysis, the alkoxy group leaves through an $S_{N} 1$ reaction producing a silanol group (Si-OH), which can form thermally and chemically stable $\mathrm{Si}-\mathrm{O}-\mathrm{M}$ bonds with the metal surface via $\mathrm{OH}$-activated metal surface $(\mathrm{M}-\mathrm{OH})$ [7]. Further condensation reactions of silanol groups form a dense network of Si-O-Si-siloxane bonds, interpenetrated with the respective organic groups. Another advantage of functional silanes (e.g, free amine group) is the ability to engage with other chemistries and further modify the surface.

The effectiveness of an orthopaedic implant in restoring damaged bone is determined by the bone-implant interaction which is characterised by the tight bond between the implant and bone surfaces, thereby minimising the risk of implant loosening over time [8]. In order to enhance the biological acceptance of orthopaedic implants, functionalisation of extracellular matrix (ECM) components has been widely employed. Hyaluronic acid (HA) is one of the major components of the extracellular matrix which is involved in cell adhesion, proliferation and differentiation. In previous studies, HA immobilised on the substrates (e.g., titanium) has not been reported as an effective inducer of osteoblastic activity [9, 10]. Moreover, the rationality of employing HA of different molecular weights for surface modification has not yet been considered. According to Zhao et al. the osteoinductive activity of bone-related cells increases in response to the high molecular weight of HA [11]. Therefore, it is important to consider the molecular weight of HA to modify the surface for different applications.

In this study, the aim is to develop a coating system where HA is bound via silane surface to deliver a corrosion resistance and cytocompatible coating on AZ31 Mg alloy for orthopaedic applications. Therefore, the functionalisation of high molecular weight HA with silanes coated on the biodegradable AZ31 Mg alloy is proposed the first time in the present work. The silanes involve an initial MTESTEOS treatment, followed by APTES deliver an amine terminated surface which can be coupled to HA through EDC-NHS coupling reactions. The corrosion resistance of the coated surface was evaluated in HEPES modified 
DMEM. Furthermore, the effect of HA on the osteoblast cellular response was evaluated.

\section{Experimental}

\subsection{Material}

AZ31 alloy sheets were obtained from Shaanxi Taipu Rare Metal Materials Ltd, China. Methyltriethoxysilane, tetraethoxysilane, 3-aminopropyl-triethoxy silane, Hyaluronic acid from rooster comb (1-4 MDa), phosphate buffer saline (PBS), Dulbecco's Modified Eagle's Medium/Nutrient F-12 Ham (DMEM/F-12), fetal bovine serum (FBS), Penicillinstreptomycin antibiotics, phosphatase substrate and bisBenzimide H 33342 trihydrochloride, NHS (N-hydroxysuccinimide), EDC (1-ethyl-3-(3-dimethylaminopropyl) carbodiimide) and MES (2-(N-morpholino)ethanesulfonic acid) buffer were purchased from Sigma Aldrich.

\subsection{Surface modifications of AZ31 Mg alloy}

\subsubsection{Treatment of $\mathrm{AZ3} 1$ with $\mathrm{NaOH}$}

AZ31 Mg alloy $2 \mathrm{~mm}$ thick sheets were cut into $10 \times 10 \mathrm{~mm}$ or $20 \times 20 \mathrm{~mm}$ pieces and polished progressively by finer $\mathrm{SiC}$ paper from 400 to 1200 grit. The samples were cleaned ultrasonically in acetone and immersed in $5 \mathrm{~N} \mathrm{NaOH}$ for $2 \mathrm{~h}$ at $60{ }^{\circ} \mathrm{C}$ and then cleaned with deionised water [12]. These hydroxide- treated AZ31 are referred to as AZ31-OH.

\subsubsection{Preparation of the hyaluronic acid functionalised silane coating on AZ31 Mg alloy}

The MTES and TEOS sols were prepared in the ethanol solution containing $0.04 \mathrm{~N}$ nitric acid as a catalyst. A molar ratio of MTES/TEOS of 2/1 was used with $R=2.36$, where $\mathrm{R}=\left[\mathrm{H}_{2} \mathrm{O}\right] /[\mathrm{MTES}+\mathrm{TEOS}]$. The APTES sol was prepared by using $400 \mathrm{mM}$ APTES in ethanol solution with $\mathrm{R}=4.5$ $\left(\mathrm{R}=\left[\mathrm{H}_{2} \mathrm{O}\right] /[\right.$ APTES] $)$. The AZ31-OH substrates were dipcoated sequentially in MTES-TEOS (AZ31-MT) and APTES sol-gels and cured at $120^{\circ} \mathrm{C}$ for $1 \mathrm{~h}$ at each step to achieve the amine-terminated AZ31-MT-A substrates.

Furthermore, high molecular weight HA was functionalised onto AZ31-MT-A by a carbodiimide-mediated coupling reaction as detailed in the previous study [13]. The final concentrations of HA used to functionalised with AZ31-MT-A samples are 1 and $2 \mathrm{mg} \cdot \mathrm{ml}^{-1}$ with the resultant samples are denoted as AZ31-MT-A-HA1 and AZ31-MTA-HA2 respectively. The effective concentration of HA immobilised on the surface was determined by the MorganElson fluorometric enzyme assay [14]. The AZ31-MT-AHA1 and AZ31-MT-A-HA2 substrates were exposed to the hyaluronidase enzyme for an appropriate time at $37.5^{\circ} \mathrm{C}$. Then, the enzyme solution was collected and incubated in a boiling water bath for $5 \mathrm{~min}$ to inactivate the enzyme. After cooling to room temperature, $25 \mu \mathrm{l}$ of tetraborate reagent $\left(0.8 \mathrm{M} \mathrm{K}_{2} \mathrm{~B}_{4} \mathrm{O}_{7} .4 \mathrm{H}_{2} \mathrm{O}\right)$ was added and incubated for $3 \mathrm{~min}$ in a boiling water bath to start the Morgan- Elson fluorometric reaction. After cooling to room temperature, $0.75 \mathrm{ml}$ of $10 \%$ w/v DMAB (p-dimethylaminobenzaldehyde) reagent was added and incubated for $20 \mathrm{~min}$ at $37.5^{\circ} \mathrm{C}$. The release of $\mathrm{N}$ acetyl glucosamine was measured by fluorescence (Ex/Em: $545 / 604 \mathrm{~nm}$ ) while the concentration of HA functionalised was determined by using a standard curve of HA.

\subsection{Characterisation of AZ31 modified samples}

All modified (AZ31-OH, AZ31-MT, AZ31-MT-A AZ31MT-A-HA1 and AZ31-MT-A-HA2) and bare AZ31 substrates were investigated using attenuated total reflection infrared spectroscopy (ATR-IR) (Perkin Elmer spotlight $400 \mathrm{~N}$, USA). The wettability of the samples was determined by static water contact angle measurements (FTÅ-200 system, UK). The surface morphology of coatings was studied by scanning electron microscope (Hitachi SU-70 FESEM (YK) with EDX and WDX elemental analysis) operating at $5.0 \mathrm{kV}$ and an atomic force microscope (AFM, Asylum MFP-3D-BIO, USA). The chemical compositions of the coatings were studied using energydispersive X-ray spectroscopy (EDX, Oxford Instruments).

\subsubsection{Electrochemical corrosion measurements}

All in vitro $\mathrm{Mg}$ alloy degradation tests were performed at $37 \pm 0.5^{\circ} \mathrm{C}$ in HEPES-buffered DMEM (15 mM HEPES). Electrochemical measurements were carried out by using Solartron 1255B potentiostat and 1287 frequency analyser equipped with a standard three-electrode setup. The cell comprised a saturated calomel electrode (SCE) as the reference electrode, a platinum mesh auxiliary electrode and the test sample (with $1 \mathrm{~cm}^{2}$ exposed area) as the working electrode. Electrochemical impedance spectra (EIS) of all samples were collected over a frequency range of $100 \mathrm{KHz}$ to $0.01 \mathrm{~Hz}$ at the measured open circuit potential with $\mathrm{AC}$ amplitude of $10 \mathrm{mV}$. A stable open circuit potential was established prior to conducting the electrochemical measurements.

\subsubsection{Immersion experiment, $\mathrm{pH}$ changes and $\mathrm{H}_{2}$ gas evolution measurement}

In order to determine the corrosion rate of the samples in the simulated medium, the silane coated and uncoated AZ31 substrates were immersed in HEPES buffered DMEM with a surface-to-volume ratio of $20 \mathrm{ml} . \mathrm{cm}^{-2}$ at 37 
Fig. 1 ATR-IR of spectra different substrates

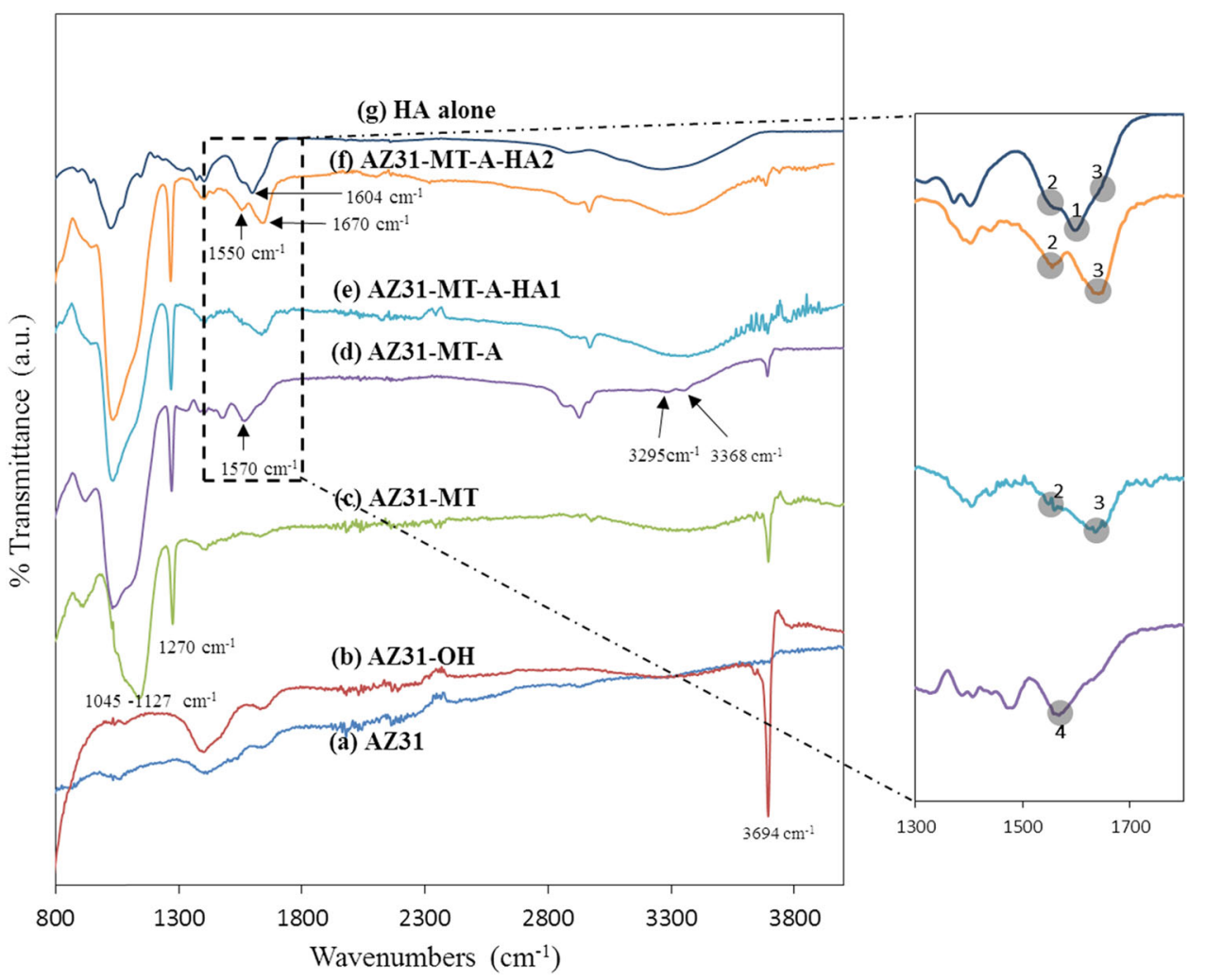

$\pm 0.5^{\circ} \mathrm{C}$ for 21 days [15]. The $\mathrm{pH}$ value (Thermo-scientific Orion 3 Star $\mathrm{pH}$ meter) of DMEM was monitored for 21 days. The release of $\mathrm{Mg}^{2+}$ ions in DMEM during this period was determined by an inductively coupled plasma optical emission spectrometer (Varian Liberty150, ICPOES) [12]. The immersion medium was refreshed every second day to maintain a continuous challenge to the sample surface. The changes in the surface morphology and the composition of the coated AZ31-MT-A substrate after 7, 14 and 21 days were determined by SEM-EDX.

The rate of $\mathrm{H}_{2}$ gas release was monitored by placing the samples in HEPES buffered DMEM at $37 \pm 0.5^{\circ} \mathrm{C}$ under an inverted funnel connected to graduated burette and measuring the medium level within the burette for upto 10 days. The ratio of the surface area-to-volume of DMEM was kept constant at $10 \mathrm{~cm}^{2} . \mathrm{L}^{-1}[16]$.

\subsection{Cytocompatibility}

\subsubsection{Live/dead cell staining}

The murine osteoblast cell line (MC3T3-E1) (ATCC ${ }^{\circledR}$ CRL$2593^{\mathrm{TM}}$, LGC standard) was used for cytocompatibility studies. Cells were cultured under $37{ }^{\circ} \mathrm{C}, 5 \% \mathrm{CO}_{2}$ and $95 \%$ relative humidity in DMEM/F-12 containing $10 \%$ FBS and $1 \%$ penicillin and streptomycin. Cells were seeded $\left(10^{4}\right.$ cells. $\mathrm{cm}^{-2}$ ) on HA functionalised AZ31-MT-A coated and uncoated AZ31 substrates and maintained in complete
DMEM/F12 $\left(1 \mathrm{~cm}^{2}=1.25 \mathrm{ml}\right)$ in accordance to ISO 10993 12 [17] for 1 and 3 days before the live-dead staining was performed. Calcein AM (eBioscience 65-0853) and propidium iodide (eBioscience 00-6990) were dissolved in DMSO $(1 \mathrm{mg} / \mathrm{ml})$ and used at 1:500 ratio in complete DMEM/F12. Cells cultured on the experimental substrates $(10 \times 10 \mathrm{~mm})$ were washed with PBS and incubated with fluorescent dyes for $20 \mathrm{~min}$ at $37^{\circ} \mathrm{C}$. After incubation cells were rinsed with PBS and imaged using an Olympus IX83 epifluorescence microscope fitted with a 10X objective.

\subsubsection{Cellular morphology imaging by SEM}

The morphology of osteoblast cells seeded at a density of $10^{4}$ cells. $\mathrm{cm}^{-2}$ on the experimental substrates was observed after 1 and 3 days of culture [17]. Cell culture conditions were maintained as described earlier. After incubation, the substrates were washed with PBS, fixed with $4 \%$ v/v formaldehyde and dehydrated in alcohol gradients. The substrates were sputter coated with Au-Pd and observed by SEM.

\subsubsection{DNA quantification}

The total cellular DNA content levels of MC3T3E1 osteoblast cells was determined as a measure of cell proliferation [12]. Cells were cultured on the coated and uncoated AZ31 substrates at a density of $2.5 \times 10^{4}$ cells.cm ${ }^{-2}$ for 3,7 

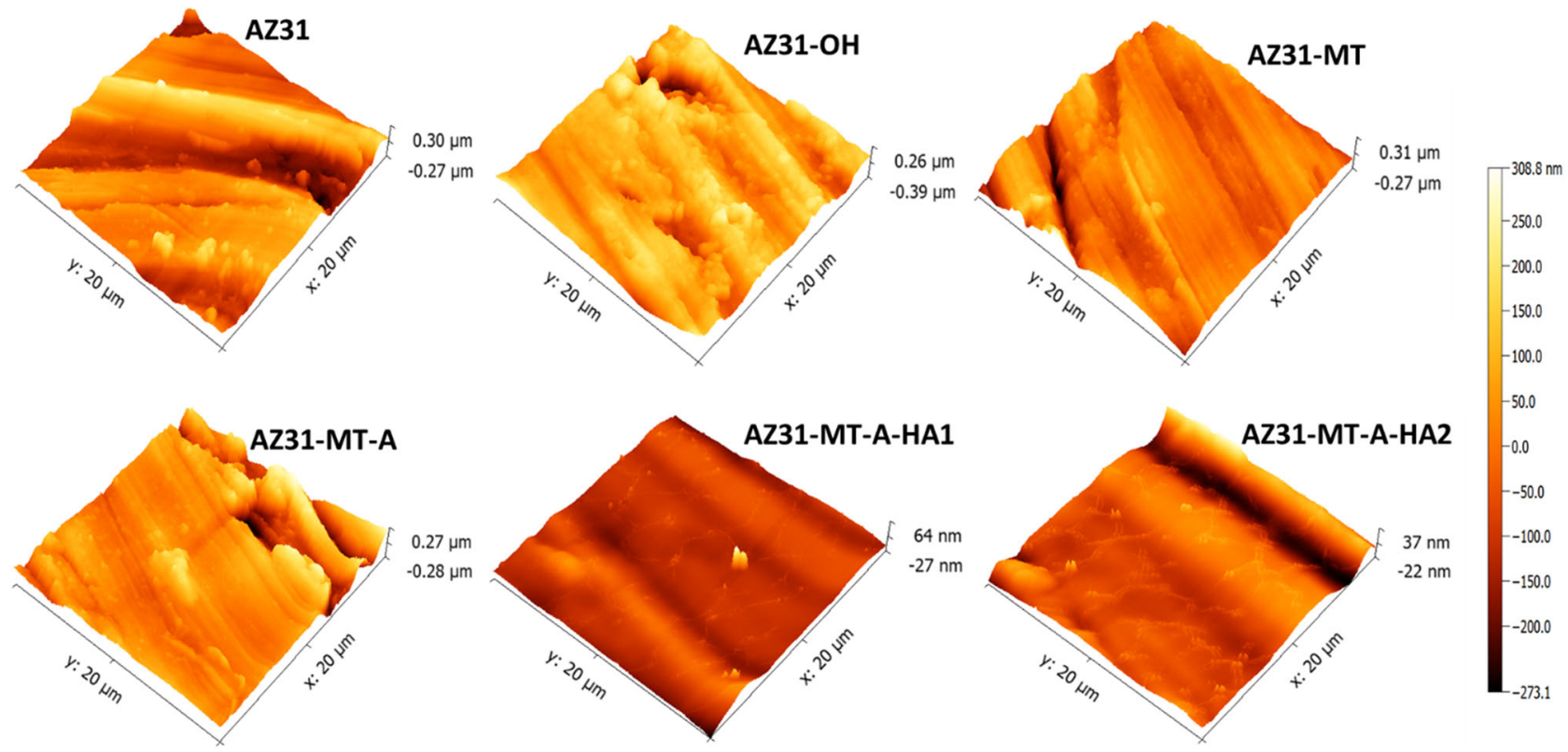

Fig. 2 AFM images of unmodified and modified substrates

and 14 days in a differentiation medium. The differentiation medium was prepared by adding $50 \mu \mathrm{M}$ ascorbic acid, 100 $\mathrm{nM}$ dexamethasone and $10 \mathrm{mM} \beta$-glycerophosphate in F12/ DMEM [12]. After 3, 7 and 14 days of culture, substrates were washed with PBS and the cells were lysed using cell lysis buffer. The fluorescent dye, Hoechst 33258 (SigmaAldrich) was used to quantify the cellular DNA according to the manufacturer's instructions (DNA quantification kit, Sigma-Aldrich).

\subsubsection{Alkaline phosphatase (ALP) assay}

The differentiation of osteoblast cells was studied by measuring intracellular ALP activity using a para-nitrophenyl phosphate substrate (p-NPP, Sigma) [18]. After 3, 7 and 14 days the cells were cultured in the differentiation medium, and the cell lysate was incubated with p-NPP for $1 \mathrm{~h}$ at $37^{\circ} \mathrm{C}$. The enzymatic reaction was terminated by adding 1 $\mathrm{N} \mathrm{NaOH}$ and the ALP activity was measured by the UV absorbance of para-nitrophenol (p-NP) at $410 \mathrm{~nm}$. A standard curve of different $\mathrm{p}-\mathrm{NP}$ concentrations was prepared by diluting in $0.02 \mathrm{~N} \mathrm{NaOH}$. The p-NP was normalised against total protein concentration. The total protein content was determined by using the bicinchoninic acid protein assay kit (Sigma-Aldrich).

\subsection{Statistical analysis}

All of the experiments were conducted in triplicate. All data are expressed as mean $\pm \mathrm{SD}$. The differences between the groups were analysed using one-way analysis of variance
(ANOVA) followed by post hoc Tukey test. Statistically significance was considered at $p<0.05$.

\section{Results and discussion}

\subsection{Characterisation of AZ31 modified surface}

In order to bond with the silane coating, the AZ31 surface was passivated with $\mathrm{NaOH}$ to produce surface hydroxy groups. The AZ31-OH was sequentially treated with hydrolysed MTES-TEOS and APTES sols to prepare a two-layer silane coating through siloxane bond formation. Following exposure of the MTES-TEOS sol, the ATR-IR spectrum (Fig. 1) shows bands around 3694, 1045-1127 and $1270 \mathrm{~cm}^{-1}$ which are attributed to $-\mathrm{OH}$ groups (Fig. 1b), Si-O asymmetric stretching of $-\mathrm{Si}-\mathrm{O}-\mathrm{Si}-$ and $\mathrm{Si}-\mathrm{CH}_{3}$ (Fig. 1c) of MTES respectively [7]. Subsequent coating with APTES increases the intensity of -Si-O-Si- band with the emergence of new bands at 1570, 3295 and $3368 \mathrm{~cm}^{-1}$ (Fig. 1d) which are associated with asymmetric and symmetric stretching modes of $\mathrm{NH}_{2}$ group, respectively [7, 19]. The amino silane surface was then functionalised with HA through EDC-NHS coupling reactions at increasing concentrations. The effective concentrations of the bound HA obtained from Morgan-Elson assay for AZ31-MT-A-HA1 and AZ31-MT-A-HA2 substrates were found to be $45.45 \pm$ $0.05 \mu \mathrm{g} . \mathrm{cm}^{2}$ and $66.77 \pm 0.25 \mu \mathrm{g} . \mathrm{cm}^{2}$ respectively. The amide I (a shoulder band), amide-II band and stretching vibrations of carboxylate $\mathrm{C}=\mathrm{O}$ of $\mathrm{HA}$ are indicated at $1670 \mathrm{~cm}^{-1}$ (Figs. 1g-3), $1540 \mathrm{~cm}^{-1}$ (Figs. 1g-2) and 
Fig. 3 Bode plots of (a) AZ31 alone, AZ3-OH, AZ31-MT, AZ31-MT-A, AZ31-MT-AHA1 and AZ31-MT-A-HA2 surfaces, and (b) equivalent circuits used to fit experimental data; coated and uncoated (AZ31 and AZ31-OH substrates)
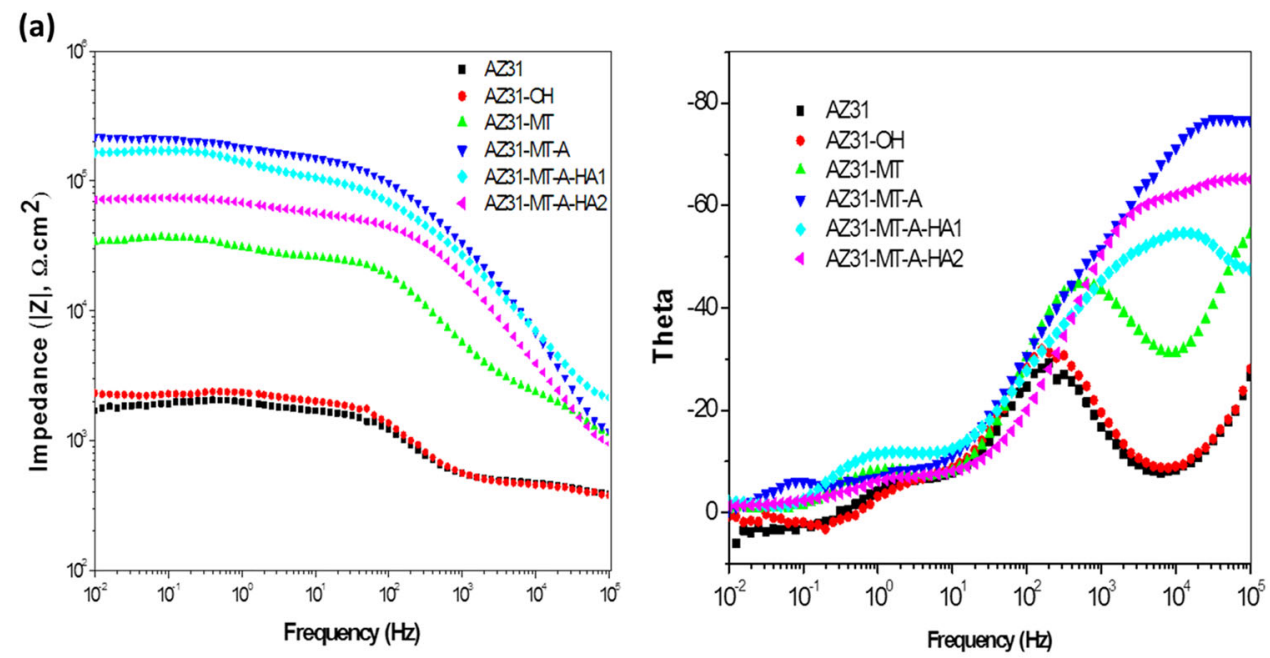

(b)

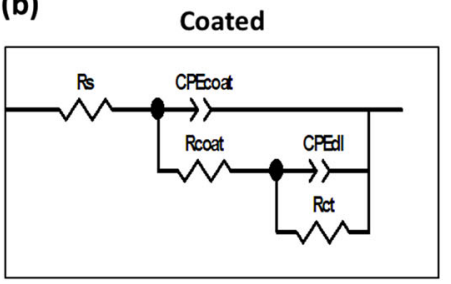

$1604 \mathrm{~cm}^{-1}$ (Figs. 1g-1) respectively [20]. With the HA functionalisation, the bands of amide I (Figs. 1f-3 and 1-e3) and amide II bands (Figs. 1f-2, 1e-2) can be observed prominently (showed in the magnified Figure). Moreover, the carbonyl HA band (Figs. 1g-1) and APTES amine band (Figs. 1d-4) cannot be observed in the resultant spectra indicating successful coupling at the surface.

Furthermore, AFM was used to observe the topography of surface modifications as shown in Fig. 2. The surface roughness $\left(R_{a}\right)$ of the $\mathrm{AZ31}$ and $\mathrm{AZ31-OH}$ substrates is $74.5 \pm 8.2 \mathrm{~nm}$ and $62.2 \pm 3.9 \mathrm{~nm}$, respectively. The $R_{a}$ values of AZ31-MT and AZ31-MT-A coated surface were found to be $43.1 \pm 0.5 \mathrm{~nm}$ and $45.7 \pm 0.82 \mathrm{~nm}$ respectively. The $R_{a}$ values then decreased considerably for AZ31-MTA-HA1 and AZ31-MT-A-HA2 substrates to $4.86 \pm 0.07 \mathrm{~nm}$ and $7.84 \pm 0.39 \mathrm{~nm}$, respectively, significantly lower than the bare AZ31 alloy alone. The fibrils pattern of HA can be observed which likely results from drying induced aggregation of HA chains [21].

The effect of surface modifications also changes the wettability of the surfaces as shown in Fig. S1. Compared to the AZ31 alloy, the water contact angle showed significant changes after every step of modification (passivation treatment and silane). After HA functionalisation, the AZ31-MT-A-HA1 and AZ31-MT-A-HA2 showed a significant decrease in contact angle having values $43.4^{\circ} \pm 1.4$ and $41.3^{\circ} \pm 6.3$ respectively when compared to the AZ31 alone.

\subsection{In vitro corrosion and degradation studies}

\subsubsection{Electrochemical impedance spectroscopy}

The EIS data for uncoated and coated AZ31 substrates are shown in Fig. 3a. The impedance $\left(|\mathrm{Z}|_{0.01 \mathrm{~Hz}}\right)$ increased gradually with every step of the silane coating. When compared to the AZ31 alloy, the impedance of AZ31-MT-A increased by nearly two orders of magnitude, indicating an effective protection of AZ31 alloy. Although $|Z|_{0.01} \mathrm{~Hz}$ decreased for AZ31-MT-A-HA1 and AZ31-MT-A-HA2 substrates, it is still higher than the AZ31 substrate. The analysis of EIS spectra was performed based on the proposed electrical equivalent circuits (EEC) as depicted in Fig. $3 \mathrm{~b}$ which fit best with the experimental data and parameters are given in Table 1.

Bodes phase angle plots for all coated substrates are characterised by high and low frequencies time constants which correspond to the response of charge transfer and mass diffusion-controlled reactions respectively. The AZ31 and AZ31-OH substrates are also characterised by two capacitive responses at higher and medium frequencies with an additional inductance at low frequency $(<0.1 \mathrm{~Hz})$. The inductance is related to adsorbed intermediate species during corrosion $[22,23]$. The EEC consists of a solution resistance $R_{s}$, a constant phase element of electrolyte/coating layer $\left(\mathrm{CPE}_{\text {coat }}\left(\mathrm{Q}_{\text {coat }}\right)\right)$ and a double electrical layer presence in the metal/coating $\left(\mathrm{CPE}_{\mathrm{dl}}\left(\mathrm{Q}_{\mathrm{dl}}\right)\right)$ interfaces (as 
(a)
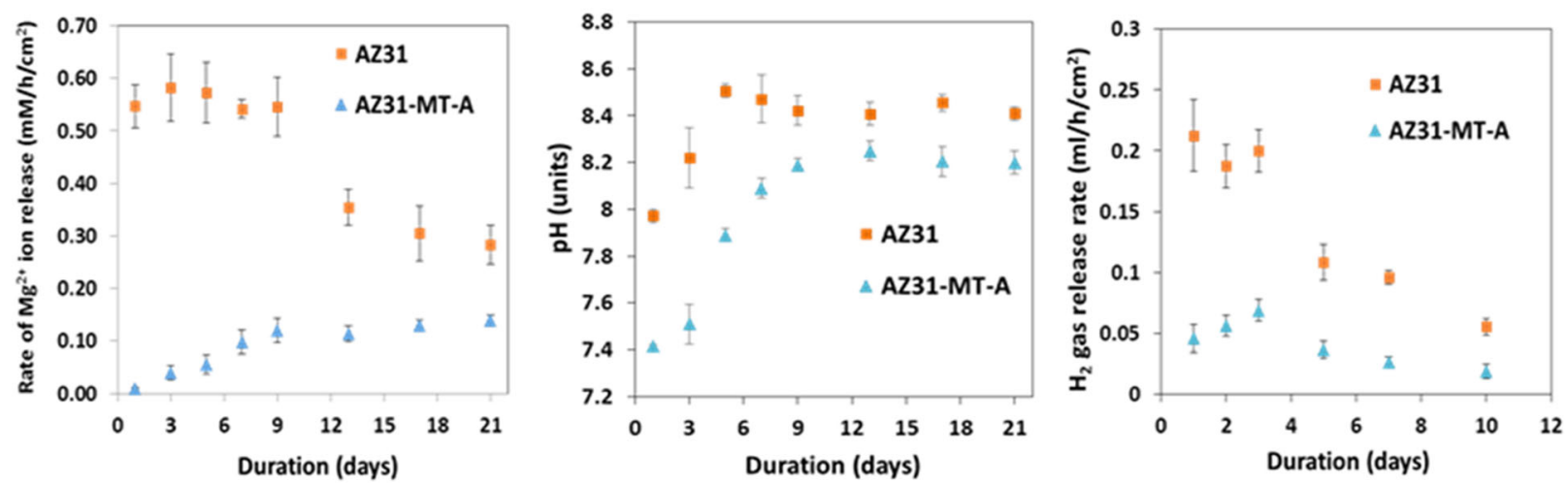

(b)
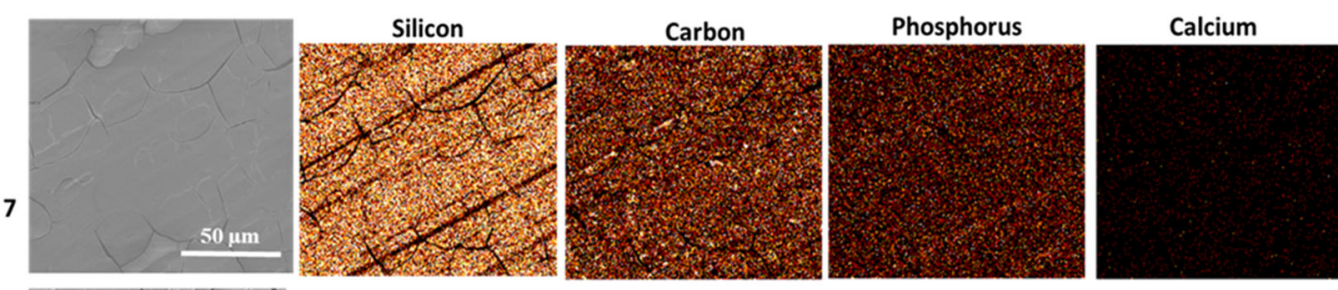

\begin{tabular}{|c|c|}
\hline Elements & Weight $\%$ \\
\hline $\mathrm{C} \mathrm{K}$ & $13.70 \pm 0.74$ \\
\hline $\mathrm{O} \mathrm{K}$ & $45.51 \pm 1.26$ \\
\hline $\mathrm{Mg} \mathrm{K}$ & $2.51 \pm 0.01$ \\
\hline $\mathrm{Si} \mathrm{K}$ & $35.21 \pm 1.61$ \\
\hline $\mathrm{P} \mathrm{K}$ & $2.37 \pm 0.05$ \\
\hline $\mathrm{Ca} \mathrm{K}$ & $0.68 \pm 0.12$ \\
\hline
\end{tabular}
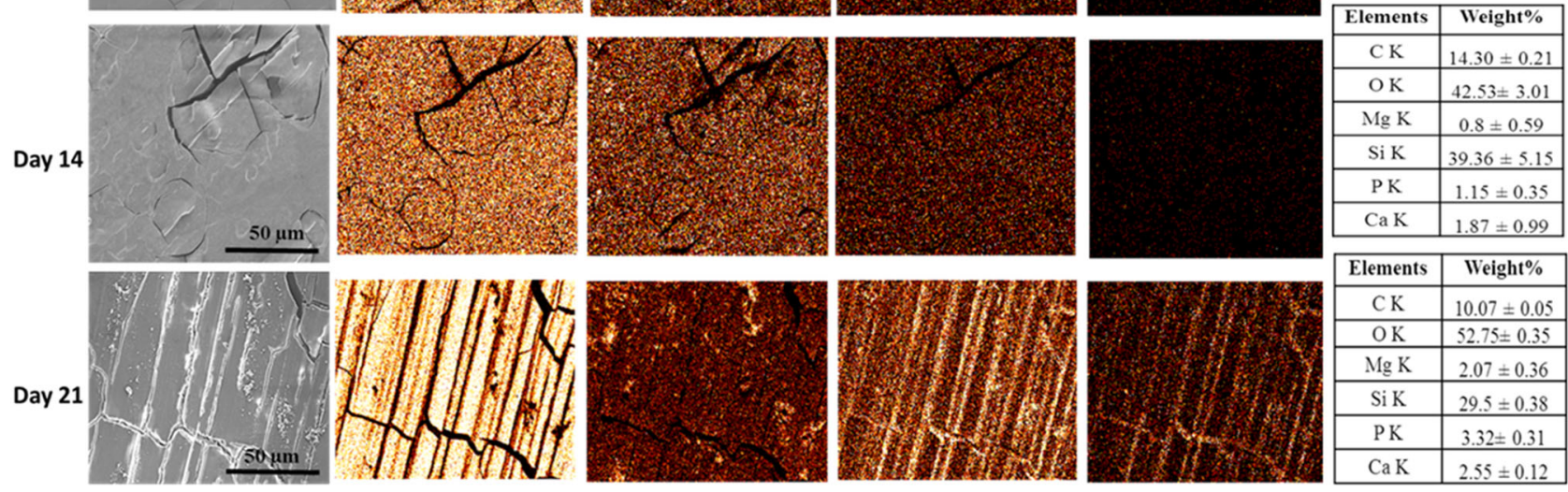

\begin{tabular}{|c|c|}
\hline Elements & Weight\% \\
\hline $\mathrm{C} \mathrm{K}$ & $10.07 \pm 0.05$ \\
\hline $\mathrm{O} \mathrm{K}$ & $52.75 \pm 0.35$ \\
\hline $\mathrm{Mg} \mathrm{K}$ & $2.07 \pm 0.36$ \\
\hline $\mathrm{Si} \mathrm{K}$ & $29.5 \pm 0.38$ \\
\hline $\mathrm{P} \mathrm{K}$ & $3.32 \pm 0.31$ \\
\hline $\mathrm{Ca} \mathrm{K}$ & $2.55 \pm 0.12$ \\
\hline
\end{tabular}

Fig. 4 a ICP-OES measurement of $\mathrm{Mg}$ ions release rate, $\mathrm{pH}$ change and rate of $\mathrm{H}_{2}$ gas release from AZ31 and AZ31-MT-A over a period of 21 and 10 days respectively, and $\mathbf{b}$ electron micrograph and EDX mapping of AZ31-MT-A substrate immersed in HEPES buffered DMEM for 7, 14 and 21 days

substantially decreased. This improved corrosion resistance of AZ31-MT-A substrate in buffered DMEM can be attributed to physical barrier performance of the siloxane coating network to electrolyte penetration [12]. This results in the retarding of anodic dissolution reactions and preventing the mass transport of $\mathrm{Mg}^{2+}$ ions and $\mathrm{H}_{2}$ gas release $[7,12]$. In a previous report, $\gamma$-APS-BTSE treated AZ31 alloy showed lower corrosion resistance than the present AZ31-MT-A model [25]. In comparison to the AZ31-MT-A substrate, immobilisation of HA on AZ31-MT-A reduced the corrosion resistance of AZ31 Mg alloy in a concentration-dependent manner (Table 1). The corrosion resistance of AZ31-MT-A-HA1 was found to be greater than the AZ31-MT-A-HA2 substrate. This may be due to the silane coating saturated with the electrolyte (MES buffer, $\mathrm{pH}$ 5.5) during the covalent coupling reaction [25]. This hinders the physical barrier function to resist electrolyte that the corrosion activity of the AZ31-MT-A substrate is 
Table 1 Parameters of uncoated and coated substrates calculated from the fitting of the experimental impedance spectra

\begin{tabular}{lllllll}
\hline Substrates & AZ31 & AZ31-OH & AZ31-MT & $\begin{array}{l}\text { AZ31-MT- } \\
\text { A }\end{array}$ & $\begin{array}{l}\text { AZ31-MT-A- } \\
\text { HA1 }\end{array}$ & $\begin{array}{l}\text { AZ31-MT-A- } \\
\text { HA2 }\end{array}$ \\
\hline $\mathrm{R}_{\mathrm{s}}\left(\Omega \cdot \mathrm{cm}^{2}\right)$ & 430 & 412 & 421.1 & 460 & 453 & 430 \\
$\mathrm{CPE}_{\mathrm{coat}} \mathrm{T}\left(\times 10^{-8} \mathrm{~F}\right.$ & 700.98 & 605.49 & 22.9269 & 4.3843 & 13.263 & 6.4226 \\
$\left.\mathrm{~cm}^{-2}\right)$ & & & & & & \\
$\mathrm{CPE}_{\mathrm{coat}} \mathrm{P}$ & 0.73861 & 0.73961 & 0.66734 & 0.73939 & 0.6323 & 0.75108 \\
$\mathrm{R}_{\mathrm{coat}}\left(\times 10^{5} \Omega . \mathrm{cm}^{2}\right)$ & 0.01435 & 0.01812 & 0.51917 & 1.5591 & 1.191 & 0.56386 \\
$\mathrm{CPE}_{\mathrm{dl}} \mathrm{T}\left(\times 10^{-6} \mathrm{~F} \mathrm{~cm}\right.$ & 124.07 & 71.72 & 7.289 & 4.5709 & 3.187 & 7.136 \\
$\mathrm{CPE}_{\mathrm{dl}} \mathrm{P}$ & & & & & & \\
$\mathrm{R}_{\mathrm{ct}}\left(\Omega \cdot \mathrm{cm}^{2}\right)$ & 0.80545 & 0.878 & 0.6794 & 0.7783 & 0.94893 & 0.87908 \\
\hline
\end{tabular}

penetration. Moreover, it is known that HA modifications have been used to impart the surface hydrophilicity due to its excellent water absorption properties [24]. Therefore, the overall increase in the corrosion resistance of AZ31-MT-AHA1 and AZ31-MT-A-HA2 substrates in comparison to the uncoated AZ31 alloy can be attributed to the corrosion resistant property of the silane component of the coating. Since HA functionalisation failed to offer any corrosion resistance, further degradation studies have been performed with the corrosion resistant AZ31-MT-A substrate with the uncoated AZ31 control.

\subsubsection{Immersion experiment, $\mathrm{pH}$ changes, and $\mathrm{H}_{2}$ evolution studies}

The $\mathrm{Mg}^{2+}$ ion release rate, $\mathrm{pH}$ change and evolution of $\mathrm{H}_{2}$ gas from the coated AZ31-MT-A and uncoated AZ31 substrates aged in HEPES buffered DMEM for 21 and 10 days respectively are shown in Fig. 4a. The AZ31MT-A substrate showed a very slow and steady release of $\mathrm{Mg}^{2+}$ ions which was $\sim 8$ fold $(0.0015 \mathrm{mM} / \mathrm{hr})$ lower as compared to uncoated AZ31 alloy $(0.013 \mathrm{mM} / \mathrm{hr})$ until day 9. For the remaining immersion period, the concentration of $\mathrm{Mg}^{2+}$ in the medium decreased thereafter to $\sim 2.5$ fold $(0.001 \mathrm{mM} / \mathrm{hr})$ lower than the uncoated AZ31 equivalents $(0.003 \mathrm{mM} / \mathrm{hr})$. This occurs mainly due to the decrease in the $\mathrm{Mg}^{2+}$ ion release rate from the uncoated AZ31 alloy. These results would confirm that the controlled degradation of coated AZ31 Mg alloy, especially during the initial period, plays an important role in maintaining physiological conditions required for bone healing.

Furthermore, the $\mathrm{pH}$ change of HEPES buffered DMEM containing uncoated and AZ31-MT-A coated substrates for 21 days showed that the AZ31-MT-A substrate was less alkaline as compared to the uncoated AZ31 alloy. The $\mathrm{pH}$ of the AZ31-MT-A substrate increases gradually until day 9 $(\mathrm{pH} 8.19 \pm 0.03)$ and remains unchanged for the remaining immersion time $(\mathrm{pH} 8.23 \pm 0.05)$, whereas AZ31 alloy exhibits a sudden increase in $\mathrm{pH}$ until day $9(\mathrm{pH} 8.43 \pm$
0.04) and then stabilises for the remaining period $(\mathrm{pH} 8.49$ \pm 0.05 ).

The rate of hydrogen gas evolution was measured from AZ31 and AZ31-MT-A substrates in HEPES buffered DMEM for 10 days. The coated substrate released less $\mathrm{H}_{2}$ gas over 10 days (from $0.045 \mathrm{ml} / \mathrm{h}$ to $0.019 \mathrm{ml} / \mathrm{h}$ ) when compared to uncoated AZ31 (from $0.22 \mathrm{ml} / \mathrm{h}$ to $0.055 \mathrm{ml} / \mathrm{h}$ ). Therefore, the coated AZ31-MT-A surface displayed superior performance (in terms of $\mathrm{pH}$ change, $\mathrm{H}_{2}$ and $\mathrm{Mg}^{2+}$ ions release) when compared to the uncoated AZ31 substrate. The difference was especially apparent during the early period of immersion.

Furthermore, the surface profile of the AZ31-MT-A substrate in buffered DMEM for different times was evaluated by SEM-EDX as illustrated in Fig. 4b. The coated substrate shows increasing crack incidence over time and evidence of corrosion debris. The EDX mapping and elements quantification (inset tables) of the aged coated substrate are rich in carbon $(\mathrm{C})$, calcium $(\mathrm{Ca})$, oxygen $(\mathrm{O})$ and phosphate $(\mathrm{P})$. A protective layer containing a relatively high content of $\mathrm{O}, \mathrm{C}, \mathrm{Ca}$ and $\mathrm{P}$ deposits were observed on the coated substrate immersed over a period of 21 days. In addition, a weak signal of Si observed on day 21, which indicates the degradation of the silane coating.

The possible reasons for the crack formation and increased deposition of corrosion by-products on the coated AZ31 alloy with the immersion time include electrolyte penetration into the coating and subsequent corrosion of the underlying passivated AZ31 alloy with the release of $\mathrm{Mg}^{2+}$ ions at the site of corrosion [26]. This results in the degradation of the silane coating over a period of time. However, the complete degradation of the silane coating has not been observed (Si EDX mapping), reflecting the stability of the coating. Generally, hydroxide passivated AZ31 surface is unable to provide long-term corrosion resistance in the chloride-rich environment [27]. This leads to the efflux of $\mathrm{Mg}^{2+}$ ions from the cracks which react with water to form an alkaline environment and produces $\mathrm{Mg}$ $(\mathrm{OH})_{2}$ and $\mathrm{H}_{2}$ gas [5]. The overall (anodic and cathodic) 
Fig. 5 a Fluorescence microscopy images of live-dead MC3T3E1 cells and b SEM morphology of fixed MC3T3E1 osteoblast cells attached on AZ31, AZ31-MT-A-HA1, and AZ31-MT-A-HA2 for day 1 and 3 . White arrows are indicating osteoblast cells
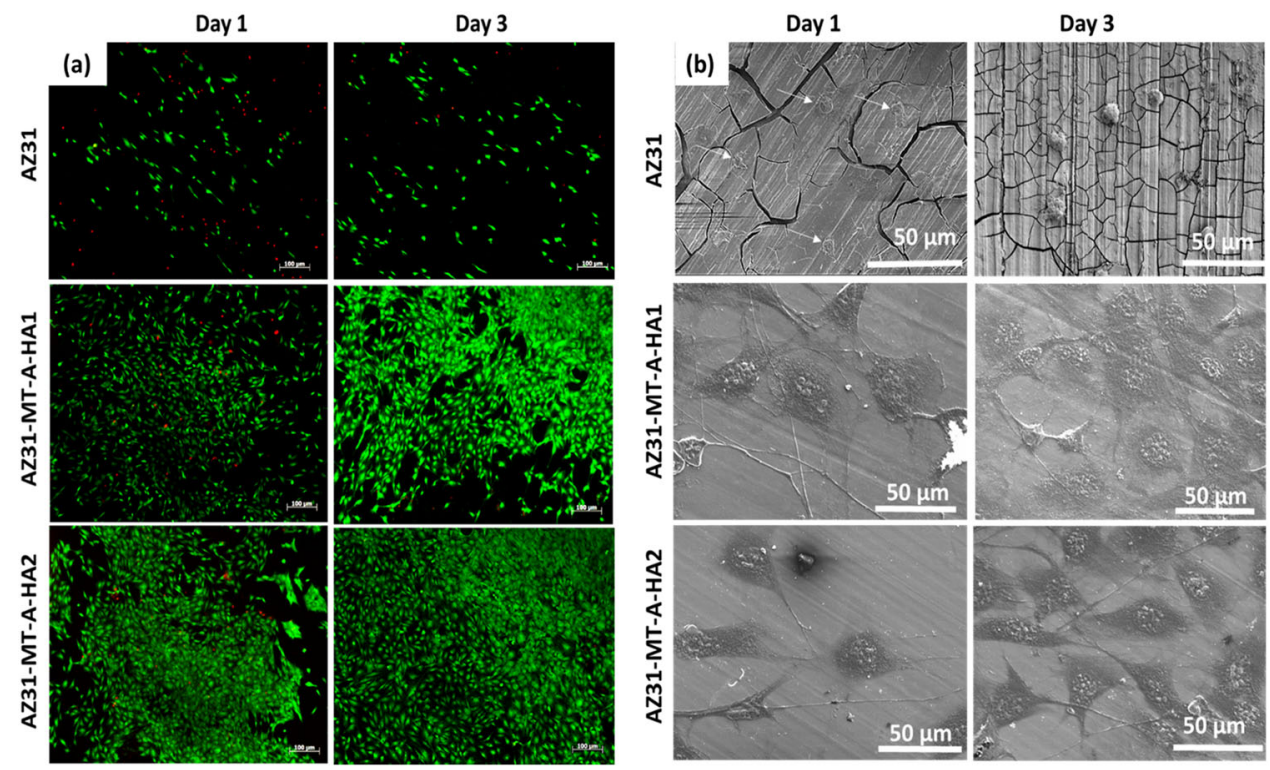

Fig. 6 a Quantification of total DNA content and $\mathbf{b}$ ALP activity of osteoblast cultured on uncoated AZ31, AZ31-MT-AHA1 and AZ31-MT-A-HA2 substrates for 3, 7 and 14 days. (Statistical significance level: one way ANOVA with posthoc Tukey test with significance level of $* * p<0.01, * p<0.05$.)
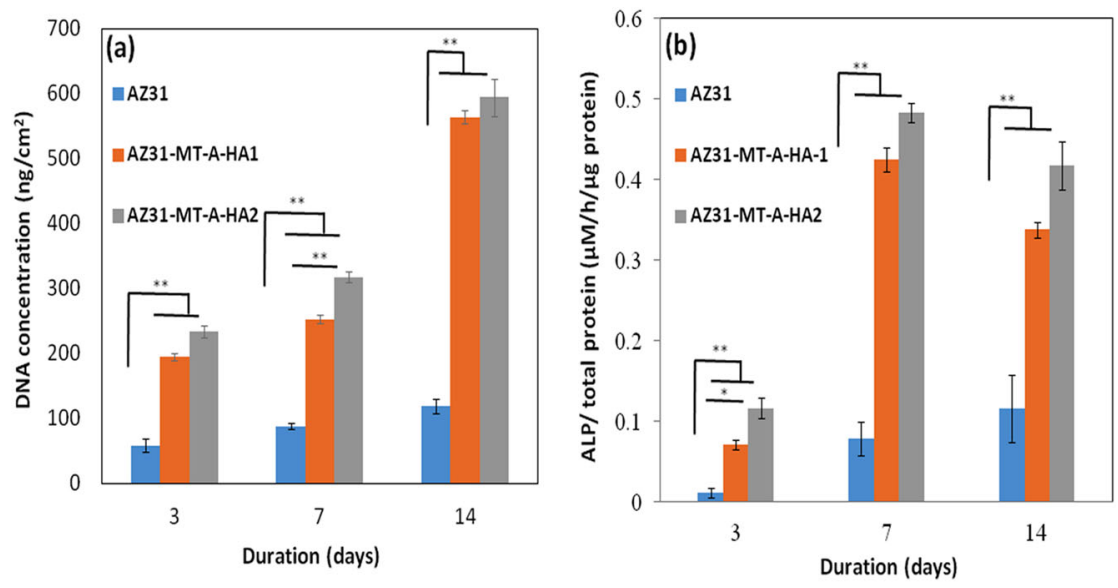

reaction as given below:

$\mathrm{Mg} \rightarrow \mathrm{Mg}^{2+}+2 \mathrm{e}^{-}($anodic reaction $)$

$2 \mathrm{H}_{2} \mathrm{O}+2 \mathrm{e}^{-} \rightarrow \mathrm{H}_{2}+2 \mathrm{OH}^{-}$(cathodic reaction)

$\mathrm{Mg}+2 \mathrm{H}_{2} \mathrm{O} \rightarrow \mathrm{H}_{2}+\mathrm{Mg}(\mathrm{OH})_{2}$ (overall reaction)

The evolution of $\mathrm{H}_{2}$ gas reduces the coating stability, which enhances electrolyte penetration through the coating, thereby promoting further corrosion. However, increases in $\mathrm{pH}$ of the medium induce the formation of a protective precipitate of Ca-P and $\mathrm{Mg}(\mathrm{OH})_{2}$ at the alloy surface, thereby reducing the degradation of $\mathrm{Mg}$ alloy [12]. This results in the reduced $\mathrm{Mg}^{2+}$ ion release rate, $\mathrm{H}_{2}$ gas release and $\mathrm{pH}$ change especially during the later stage of immersion period as observed for both coated and uncoated substrates (Fig. 4a). Previous reports also showed that the passivation layer formed during the corrosion process reduced the degradation rate of $\mathrm{Mg}$ alloys in vitro [9]. This controlled degradation of coated AZ31-MT-A substrate could be attributed to the coating stability and in situ formation of protective degradation layers, helping maintain conditions required for osteoblast cell growth.

\subsection{Cytocompatibility studies}

For cytocompatibility studies, HA was functionalised onto the AZ31-MT-A substrate to develop a biocompatible and osteoinductive surface. The live-dead staining of MC3T3E1 osteoblast cells cultured on AZ31, AZ31-MT-A-HA1 and AZ31-MT-A-HA2 substrates for day 1 and 3 can be seen in Fig. 5a. For both time points, the AZ31-MT-A-HA1 and AZ31-MT-A-HA2 substrates showed the HA concentration-dependent increase in live cell density (green fluorescence) with very few dead cells (red fluorescence) as compared to uncoated AZ31 alloy, indicating an enhanced 
cell adhesion and proliferation of osteoblast cells on HA coated AZ31-MT-A substrates.

The SEM images of the fixed osteoblast cells on HA coated AZ31-MT-A and uncoated AZ31 substrates on day 1 and 3 can be seen in Fig. 5b. The cells attached to the uncoated AZ31 substrate on day 1 and 3 are sparse and circular in shape (indicated by white arrows), indicating very poor adhesion to the substrate. On the other hand, osteoblast cells on AZ31-MT-A-HA1 and AZ31-MT-AHA2 substrates displayed more flattened spread morphology, indicating good osteoblast cell attachment. These results suggested that HA coated surfaces facilitate greater adhesion of osteoblast cells.

The total DNA content of osteoblasts cultured on uncoated and coated AZ31 substrates was quantified over a period of 14 days as shown in Fig. 6a. The low DNA content on day 3 confirmed that very few cells attached initially on the AZ31 surface. This was expected due to the rapid degradation of AZ31 alloy in HEPES-DMEM [27]. On the other hand, 4 fold increase in DNA content was observed for the HA-coated surfaces on day 3, indicating that such a coating favours osteoblast cell attachment. On HA coated surfaces, adhered cells showed significant proliferation $(p<0.01)$ between 7 and 14 days as compared to the AZ31 alone. These results were also consistent with the live-dead images of osteoblast cells. However, the proliferation of osteoblasts on AZ31-MT-A-HA2 showed an insignificant increase when compared to the AZ31-MT-AHA1 substrate at the end of the culture period.

The ALP activity of osteoblast cells cultured on AZ31 alone, AZ31-MT-A-HA1 and AZ31-MT-A-HA2 substrates for 14 days is presented in Fig. 6b. Osteoblasts cultured on HA functionalised substrates displayed significantly higher ALP expression $(p<0.01)$ when compared to the uncoated AZ31 alloy over a culture duration of 14 days. However, increase in the ALP expression is insignificant for both the HA functionalised surfaces. Therefore, the AZ31-MT-AHA1 substrate can be considered more efficient in terms of osteoblast response, as it enhanced the osteoblasts proliferation and differentiation significantly at a lower concentration of immobilised HA over uncoated AZ31 substrate. ALP is one of the most widely used early stage marker of osteoblast differentiation, which participates in ECM mineralisation [10]. HA is one of the major ECM component of many cells including osteoblast cells which regulates cell adhesion, proliferation and expression of osteoinductive factors including ALP [28]. However, very few reports evaluated cell adhesion, proliferation and differentiation ability of HA functionalisation on various metal substrates. Chua et al. showed the poor adhesion of osteoblasts on HA modified Ti samples as compared to the chitosan/HA or chitosan/HA/RGD peptides functionalised Ti surface [29]. Similarly, $\mathrm{Hu}$ et al. also reported poor adhesion and proliferation of osteoblasts on hyaluronic acid-catechol functionalised on $\mathrm{Ti}$ surface (Ti-HAC) as compared to the Ti-HAC-VEGF and Ti-CMC-VEGF [30]. However, no details about the molecular weight of HA were furnished in these studies [29, 30]. On the contrary, the present study demonstrated remarkable osteoblast cell adhesion, proliferation and differentiation on HA functionalised AZ31 substrates. Zhao et al. studied the hyaluronic acid molecular weight dependent differentiation of rBMSCs [11]. It has been reported that high molecular weight HA promoted bone-related cells proliferation and differentiation [11]. The enhanced osteogenic differentiation of osteoblast cells on HA coated AZ31 substrate could be attributed to the immobilisation of high molecular weight HA.

\section{Conclusion}

In the present work, a multilayered hyaluronic acid functionalised silane coating on AZ31 Mg alloy was fabricated successfully. Electrochemical corrosion study demonstrated that the silane component of the coating significantly improved the corrosion resistance of the AZ31-MT-A-HA1/ HA2 substrates and an overall increase in the corrosion resistance was observed for AZ31-MT-A-HA1 substrate when compared to the AZ31-MT-A-HA2 and uncoated AZ31 substrates. Furthermore, immersion studies showed that stability of the silane coating as well as in situ formations of passivation layer played an important role in the controlled degradation of the coated AZ31 Mg alloy, thereby keeping the conditions necessary for the growth of osteoblasts. Amongst HA (high molecular weight) functionalised AZ31-MT-A substrates, AZ31-MT-A-HA1 efficiently enhanced the cytocompatibility over uncoated AZ31 Mg alloy, as demonstrated by a significant increase in the proliferation and differentiation of osteoblast cells. Collectively, these results demonstrated that the proposed strategy to develop a multifunctional coating which can improve the corrosion resistance and cytocompatibility of AZ31 $\mathrm{Mg}$ alloy would be highly desirable for orthopaedic applications.

Acknowledgements The authors acknowledge Dublin Institute of Technology for the financial support through Fiosraigh Scholarship Programme 2014.

\section{Compliance with ethical standards}

Conflict of interest The authors declares that they have no conflict of interest.

\section{References}

1. Witte F, Fischer J, Nellesen J, Crostack HA, Kaese V, Pisch A et al. In vitro and in vivo corrosion measurements of magnesium 
alloys. Biomaterials. 2006;27:1013-8. https://doi.org/10.1016/j. biomaterials.2005.07.037.

2. Walker J, Shadanbaz S, Woodfield TBF, Staiger MP, Dias GJ. Magnesium biomaterials for orthopedic application: a review from a biological perspective. J Biomed Mater Res - Part B Appl Biomater. 2014;102:1316-31. https://doi.org/10.1002/jbm.b. 33113.

3. Sanchez AHM, Luthringer BJC, Feyerabend F, Willumeit R. Mg and $\mathrm{Mg}$ alloys: How comparable are in vitro and in vivo corrosion rates? A review. Acta Biomater. 2015;13:16-31. https://doi.org/ 10.1016/j.actbio.2014.11.048.

4. Xin $\mathrm{Y}, \mathrm{Hu} \mathrm{T}, \mathrm{Chu} \mathrm{PK}$. In vitro studies of biomedical magnesium alloys in a simulated physiological environment: a review. Acta Biomater. 2011;7:1452-9. https://doi.org/10.1016/j.actbio.2010. 12.004 .

5. Agarwal S, Curtin J, Duffy B, Jaiswal S. Biodegradable magnesium alloys for orthopaedic applications: a review on corrosion, biocompatibility and surface modifications. Mater Sci Eng C. 2016;68:948-63. https://doi.org/10.1016/j.msec.2016.06.020.

6. Whelan M, Cassidy J, Duffy B. Sol-gel sealing characteristics for corrosion resistance of anodised aluminium. Surf Coat Technol. 2013;235:86-96. https://doi.org/10.1016/j.surfcoat.2013.07.018.

7. Song J, Ooij WJVan. Bonding and corrosion protection mechanisms of $\gamma$-APS and BTSE silane films on aluminum substrates. J Adhes Sci Technol. 2003;17:2191-21. https://doi.org/10. 1163/156856103772150788.

8. Shalabi MM, Wolke JGC, Cuijpers VMJI, Jansen Ja. Evaluation of bone response to titanium-coated polymethyl methacrylate resin (PMMA) implants by X-ray tomography. J Mater Sci Mater Med. 2007;18:2033-9. https://doi.org/10.1007/s10856-007-3160-0.

9. Agarwal S, Morshed M, Labour MN, Hoey D, Duffy B, Curtin J et al. Enhanced corrosion protection and biocompatibility of a PLGA-silane coating on AZ31 Mg alloy for orthopaedic applications. RSC Adv. 2016;6:113871-83. https://doi.org/10.1039/ C6RA24382G.

10. Gu XN, Li N, Zheng YF, Ruan L. In vitro degradation performance and biological response of a $\mathrm{Mg}-\mathrm{Zn}-\mathrm{Zr}$ alloy. Mater Sci Eng B Solid-State Mater Adv Technol. 2011;176:1778-84. https://doi.org/10.1016/j.mseb.2011.05.032.

11. Zhao N, Wang X, Qin L, Guo Z, Li D. Effect of molecular weight and concentration of hyaluronan on cell proliferation and osteogenic differentiation in vitro. Biochem Biophys Res Commun. 2015;465:569-74. https://doi.org/10.1016/j.bbrc.2015.08.061.

12. Kunjukunju S, Roy A, Ramanathan M, Lee B, Candiello JE, Kumta PN. A layer-by-layer approach to natural polymer-derived bioactive coatings on magnesium alloys. Acta Biomater. 2013;9:8690-703. https://doi.org/10.1016/j.actbio.2013.05.013.

13. D'Sa RA, Dickinson PJ, Raj J, Pierscionek BK, Meenan BJ. Inhibition of lens epithelial cell growth via immobilisation of hyaluronic acid on atmospheric pressure plasma modified polystyrene. Soft Matter. 2011;7:608-17. https://doi.org/10.1039/ C0SM00936A.

14. Takahashi T, Ikegami-Kawai M, Okuda R, Suzuki K. A fluorimetric Morgan-Elson assay method for hyaluronidase activity. Anal Biochem. 2003;322:257-63. https://doi.org/10.1016/j.ab. 2003.08.005.

15. Homayun B, Afshar A. Microstructure, mechanical properties, corrosion behavior and cytotoxicity of $\mathrm{Mg}-\mathrm{Zn}-\mathrm{Al}-\mathrm{Ca}$ alloys as biodegradable materials. J Alloy Compd. 2014;607:1-10. https:// doi.org/10.1016/j.jallcom.2014.04.059.

16. Bobe K, Willbold E, Morgenthal I, Andersen O, Studnitzky T, Nellesen $\mathbf{J}$ et al. In vitro and in vivo evaluation of biodegradable, open-porous scaffolds made of sintered magnesium W4 short fibres. Acta Biomater. 2013;9:8611-23. https://doi.org/10.1016/j. actbio.2013.03.035.
17. Chou DT, Hong D, Saha P, Ferrero J, Lee B, Tan Z et al. In vitro and in vivo corrosion, cytocompatibility and mechanical properties of biodegradable $\mathrm{Mg}-\mathrm{Y}-\mathrm{Ca}-\mathrm{Zr}$ alloys as implant materials. Acta Biomater. 2013;9:8518-33. https://doi.org/10.1016/j.actbio. 2013.06.025.

18. Pandey M, Kapila R, Kapila S. Osteoanabolic activity of wheyderived anti-oxidative (MHIRL and YVEEL) and angiotensinconverting enzyme inhibitory (YLLF, ALPMHIR, IPA and WLAHK) bioactive peptides. Peptides. 2018;99:1-7. https://doi. org/10.1016/j.peptides.2017.11.004.

19. Majoul N, Aouida S, Bessaïs B. Progress of porous silicon APTES-functionalization by FTIR investigations. Appl Surf Sci. 2015;331:388-91. https://doi.org/10.1016/j.apsusc.2015.01.107.

20. Almeida PV, Shahbazi M-A, Mäkilä E, Kaasalainen M, Salonen J, Hirvonen $\mathrm{J}$ et al.Amine-modified hyaluronic acid-functionalized porous silicon nanoparticles for targeting breast cancer tumors. Nanoscale. 2014;6:10377-87.https://doi.org/10.1039/c4nr02187h.

21. Liu X, Huang R, Su R, Qi W, Wang L, He Z. Grafting hyaluronic acid onto gold surface to achieve low protein fouling in surface plasmon resonance biosensors. ACS Appl Mater Interfaces. 2014;6:13034-42. https://doi.org/10.1021/am502921z.

22. King AD, Birbilis N, Scully JR. Accurate electrochemical measurement of magnesium corrosion rates; a combined impedance, mass-loss and hydrogen collection study. Electrochim Acta. 2014;121:394-406. https://doi.org/10.1016/j.electacta.2013.12. 124.

23. Srinivasan A, Shin KS, Rajendran N. Influence of bicarbonate concentration on the conversion layer formation onto AZ31 magnesium alloy and its electrochemical corrosion behaviour in simulated body fluid. RSC Adv. 2016;6:49910-22. https://doi.org/ 10.1039/C6RA08478H.

24. Weeks A, Boone A, Luensmann D, Jones L, Sheardown H. The effects of hyaluronic acid incorporated as a wetting agent on lysozyme denaturation in model contact lens materials. J Biomater Appl. 2012;28:323-33. https://doi.org/10.1177/ 0885328212446936

25. Liu X, Yue Z, Romeo T, Weber J, Scheuermann T, Moulton S. et al. Biofunctionalized anti-corrosive silane coatings for magnesium alloys. Acta Biomater. 2013;9:8671-7. https://doi.org/10. 1016/j.actbio.2012.12.025.

26. Jamesh M, Kumar S, Narayanan TSNS. Electrodeposition of hydroxyapatite coating on magnesium for biomedical applications. J Coat Technol Res. 2012;9:495-502. https://doi.org/10. 1007/s11998-011-9382-6.

27. Dezfuli SN, Huan Z, Mol JMC, Leeflang MA, Chang J, Zhou J. Influence of HEPES buffer on the local $\mathrm{pH}$ and formation of surface layer during in vitro degradation tests of magnesium in DMEM. Prog Nat Sci Mater Int. 2014;24:531-8. https://doi.org/ 10.1016/j.pnsc.2014.08.009.

28. Kawano M, Ariyoshi W, Iwanaga K, Okinaga T, Habu M, Yoshioka I et al. Mechanism involved in enhancement of osteoblast differentiation by hyaluronic acid. Biochem Biophys Res Commun. 2011;405:575-80. https://doi.org/10.1016/j.bbrc.2011. 01.071.

29. Chua PH, Neoh KG, Kang ET, Wang W. Surface functionalization of titanium with hyaluronic acid/chitosan polyelectrolyte multilayers and RGD for promoting osteoblast functions and inhibiting bacterial adhesion. Biomaterials. 2008;29:1412-21. https://doi.org/10.1016/j.biomaterials.2007.12.019.

30. Hu X, Neoh KG, Shi Z, Kang ET, Poh C, Wang W. An in vitro assessment of titanium functionalized with polysaccharides conjugated with vascular endothelial growth factor for enhanced osseointegration and inhibition of bacterial adhesion. Biomaterials. 2010;31:8854-63. https://doi.org/10.1016/j.biomaterials. 2010.08.006 